\title{
Outcome of 102 patients under 5 years of age with Hodgkin lymphoma
}

\author{
Asuman N. Karhann, M.D., Prof. Ali Varan ${ }^{b}$,Prof. Canan Akyüz $z^{b}$,Prof. Burça Aydın \\ Prof. Bilgehan Yalçın ${ }^{b}$, Prof. Tezer Kutluk $k^{b}$ and Prof. Münevver Büyükpamukçu ${ }^{b}$
}

\begin{abstract}
Background. Hodgkin's lymphoma (HL) is one of the most curable pediatric cancers, however it is rare among children under five years of age and prognostic factors for survival rate are still unknown due to low frequency in this age group. Objectives. The aim of this study was to evaluate clinical characteristics, treatment regimens, and outcome of patients under five years of age with HL.

Methods. Patients diagnosed with HL between 1972 and 2013 were retrospectively evaluated. All patients were treated with chemotherapy with or without radiotherapy.

Results. There were 102 patients with a median age of 4 years (range: 2 to 4.9 ). The median followup time was 13 years. Twenty-three patients had B symptoms, 15 patients had 'bulky disease' and the most common stages were stage I and II. Overall survival (OS) rates were significantly different according to the stage of the cancer $(p=0.008)$. Although there were no statistically significant differences; the positivity of 'bulky disease' and B symptoms were associated with poor prognosis.

Conclusion. Our single-center study included the largest number of patients under five years of age with HL. The stage was the main predictor for OS; on the other hand, the presence of B symptoms and bulky disease has also affected the prognosis. Key words: Hodgkin's lymphoma, adolescent, young adults, child.

http:/ / dx.doi.org/10.5546/ aap.2019.eng.e459

To cite: Karhan AN, Varan A, Akyüz C, Aydin B, et al. Outcome of 102 patients under 5 years of age with Hodgkin lymphoma. Arch Argent Pediatr 2019; 117(5):e459-e465.
\end{abstract}

\section{INTRODUCTION}

Hodgkin's lymphoma (HL) mainly occurs in adolescent and young adults, with less prevalence observed among children. In developed countries, the disease is very rare among children under five years of age, therefore the precise causes of HL in early childhood are still unknown. ${ }^{1}$ In this age group, poor prognostic factors such as bulky disease, B symptoms, and early stages are less common compared to adolesecent ages. All prior published studies have included limited numbers of patients within this age group. ${ }^{2,3}$ There is a recently published study that included one of the larger numbers of patients from Italy that evaluated 135 patients with HL. ${ }^{4}$

The treatment for children with HL generally requires a combination of risk-adapted multiagent chemotherapy with low-dose involved field radiotherapy (IFRT). This combined therapy aims to reduce the occurrence of therapyrelated toxicities, including acute myeloid leukemia, infertility, and cardiotoxicity. ${ }^{3,5,6}$ The aim of this study was to evaluate clinical characteristics, treatment regimens, and the outcome of patients under five years of age with HL.

\section{MATERIALS AND METHODS}

The patients who were diagnosed and treated with HL under 5 years of age in Hacettepe University, Department of Pediatric Oncology between 1972 and 2013 were enrolled in the study.

The patients were assessed retrospectively based on their epidemiological, clinical, 
histopathological characteristics, B symptoms (fever, night sweats and weight loss), extranodal diseases, treatment options, complications, and outcomes. The data was collected with the approval of the Institutional Research Advisory Council.

Diagnosis of HL was made through the histopathological examination of biopsy samples from the patients. Histopathological subtypes were constituted from two main groups: classical and nodular lymphocyte predominant. Classical HL consisted of four subtypes: lymphocyte-rich, mixed cellularity, lymphocytedepleted, and nodular sclerosis.

The staging was done before the initiation of treatment according to the Ann Arbor staging criteria. Staging work-up included chest X-rays, abdominal ultrasounds, thoracic and abdominopelvic computed tomography (abdominal computed tomography includes pelvic imagings; lung computed tomography includes neck component) in addition to physical examinations and laboratory tests. During the period when advanced investigation techniques did not exist, staging was done through physical examinations, bone marrow biopsies, chest X-rays and staging laparotomies. Patients displaying B symptoms were considered having unfavorable diseases.

\section{Chemotherapy protocol}

Each patient received chemotherapy and radiotherapy; however, treatment protocols were modified over the years. In the first period, all patients regardless of their stages or histopathology were treated with 6 courses of COPP (cyclophosphamide, vincristine, procarbazine and prednisolonecyclophosphamide $600 \mathrm{mg} / \mathrm{m}^{2}$ on days 1 and 7 , intravenously, vincristine $1.4 \mathrm{mg} / \mathrm{m}^{2}$ on days 1 and 7, intravenously, prednisolone $40 \mathrm{mg} / \mathrm{m}^{2}$ peroral for 14 days, procarbazine $100 \mathrm{mg} / \mathrm{m}^{2}$ peroral for 14 days) protocol and radiotherapy.

In the second period, stage I and II patients with mixed cellularity (MC) and lymphocyte predominant forms were treated with three courses of COPP with the same doses and involved field $2250 \mathrm{cGy}$ radiotherapy. Stage I and II patients with nodular sclerosing (NS) or lymphocyte depleted histopathology were treated with three courses of ABVD (adriamycin, bleomycin, vinblastine and dacarbazineadriamycin $25 \mathrm{mg} / \mathrm{m}^{2}$ on days 1 and 14 , intravenously, bleomycin $10 \mathrm{mg} / \mathrm{m}^{2}$ on days 1 and 14, intravenously, vinblastine $6 \mathrm{mg} / \mathrm{m}^{2}$ on days 1 and 14, intravenously, dacarbazine 375 $\mathrm{mg} / \mathrm{m}^{2}$ on days 1 and 14 , intravenously) and IFRT. All stage III and IV patients were treated with three courses of COPP and involved field radiotherapy. During the third and fourth period, patients received ABVD chemotherapy and involved field radiotherapy. In those periods, early-stage patients received three courses of chemotherapy; those in advanced stages underwent a six-course sandwich chemotherapy protocol. The doses were as previously described. In the last decade, to decrease infertility risk, boys with mediastinal disease were treated with the ABVD/COPP alternating regimen that also consisted of three courses in early stages and 6 courses in advanced stages.

\section{Radiotherapy protocol}

Cobalt 60 teletherapy unit was used until 1993 and linear accelerator with 6 MV x-rays thereafter. Conventional fractionation was used using daily fractions of 150 to 180 cGy. No specific immobilization device was used during treatment. In few patients, vacuumed immobilization beds or thermoplastic immobilisation masks were used. Radiotherapy was applied as mantle, minimantle, and inverted $Y$ according to disease extension in early years. Involved field radiotherapy conforming to the regions of the Ann Arbor classification was used later. Total radiotherapy dose was decided depending on response to the chemotherapy and initial volume of the disease. Total radiotherapy doses in the first period were between 3,000 and 4,000 Gy. Total radiotherapy doses afterwards ranged between 2,000 to 2,500 cGy (Median dose: 2,250 cGy). Sedation was used during radiotherapy when needed.

Multi-agent chemotherapy, combined with low-dose IFRT, has been the main option for the treatment of patients. Patients diagnosed during 1971-1980 received COPP chemotherapy and radiotherapy. Those diagnosed during 1981-1990 as having early stages of disease and either mixed cellularity or lymphocyte predominant histopathological subtypes received a combination of three cycles of COPP and IFRT. The patients in advanced stages during the same period received the combination of three cycles of COPP and three cycles of ABVD with involved field radiotherapy. The patients in the early stages, diagnosed between 1990 and 2000 and beyond, received three cycles of ABVD, whereas those advanced stages received alternating six 
cycles of COPP / ABVD along with IFRT. The patients diagnosed in 2010 have received riskadapted combined therapy. The patients were treated according to response to PET CT after 2010.

The Statistical Package for Social Sciences (SPSS) version 18.0 was used for all statistical analysis. Event-free survival (EFS) was defined as the period from patient enrollment till the date of the first event (relapse, progression, or death from any cause) or till the date of last follow-up. OS was defined as the period from enrollment till the date of death from any cause or till the last follow-up. The trends of both OS and EFS were estimated using the Kaplan-Meier method. The log-rank test was used to compare differences in OS and EFS rates. A p-value less than or equal to 0.05 was considered statistically significant.

The ethical approval was obtained from institutinal review board, and there is no conflict of interest.

\section{RESULTS}

One hundred and two patients under five years of age were selected from 728 patients with HL $(14 \%)$ and enrolled in the trial (Figure 1). Demographic and clinical characteristics of the patients are listed in Table 1. The male-female ratio was 3.8:1. The median age was 4 years (range: 2 to 4.9 ). Forty-seven patients (46\%) were diagnosed between 1981-1990. Twenty-five patients $(25 \%)$ had stage III-IV disease, while 77 $(75 \%)$ had stage I and II disease. Twenty-two patients $(22 \%)$ displayed systemic B symptoms.

FIGURE 1. Flow chart showing the patient selection

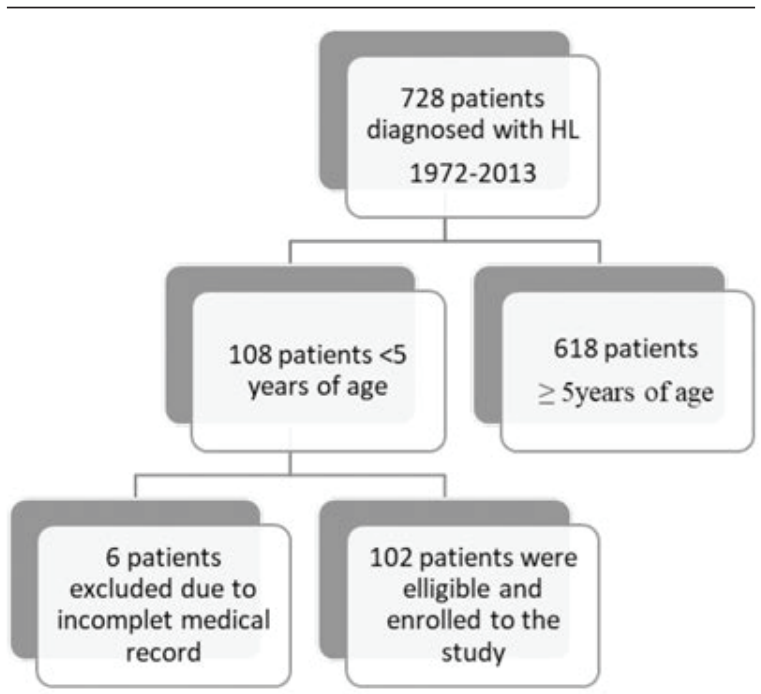

TABLE 1. Clinical characteristics of 102 patients with Hodgkin lymphoma under 5 years

\begin{tabular}{|c|c|c|}
\hline Numb & r of patients & $\%$ \\
\hline \multicolumn{3}{|l|}{ Sex } \\
\hline Male & 81 & 79.4 \\
\hline Female & 21 & 20.6 \\
\hline \multicolumn{3}{|l|}{ Diag. period } \\
\hline $1970-1980$ & 12 & 11.8 \\
\hline 1981-1990 & 47 & 46.0 \\
\hline $1991-2000$ & 29 & 28.5 \\
\hline$>2000$ & 14 & 13.7 \\
\hline \multicolumn{3}{|l|}{ Primary tumor localization } \\
\hline Cervical & 91 & 89.5 \\
\hline Axilla & 5 & 5.2 \\
\hline Abdomen & 4 & 3.5 \\
\hline Mediasten & 2 & 1.8 \\
\hline \multicolumn{3}{|l|}{ "Bulky" disease } \\
\hline Present & 15 & 14.7 \\
\hline Absent & 87 & 85.3 \\
\hline \multicolumn{3}{|l|}{ Extranodal disease } \\
\hline Present & 7 & 6.9 \\
\hline Absent & 95 & 93.1 \\
\hline \multicolumn{3}{|l|}{ B symptoms } \\
\hline Present & 23 & 22.5 \\
\hline Absent & 79 & 77.5 \\
\hline \multicolumn{3}{|l|}{ Sedimentation rate } \\
\hline High & 31 & 30.4 \\
\hline Low & 19 & 18.5 \\
\hline Unknown & 52 & 50.1 \\
\hline \multicolumn{3}{|l|}{ LDH } \\
\hline High & 31 & 30.4 \\
\hline Low & 23 & 22.5 \\
\hline Unknown & 48 & 47.1 \\
\hline \multicolumn{3}{|l|}{ Leukocytosis } \\
\hline Present & 33 & 32.4 \\
\hline Absent & 69 & 67.6 \\
\hline \multicolumn{3}{|l|}{ Anemia } \\
\hline Present & 24 & 23.5 \\
\hline Absent & 78 & 76.5 \\
\hline \multicolumn{3}{|l|}{ Stage } \\
\hline I & 45 & 44.1 \\
\hline II & 32 & 31.4 \\
\hline III & 16 & 15.7 \\
\hline IV & 9 & 8.8 \\
\hline \multicolumn{3}{|l|}{ Histopathological type } \\
\hline Mixed cellularity & 63 & 62.2 \\
\hline Lymphocyte rich & 11 & 11.1 \\
\hline Nodular sclerosis & 12 & 11.4 \\
\hline Lymphocite deplated & 3 & 2.2 \\
\hline Nodular lymphocite predominant & 2 & 2.0 \\
\hline Unclassified & 11 & 11.1 \\
\hline \multicolumn{3}{|l|}{ Chemotherapy } \\
\hline COPP & 59 & 57.7 \\
\hline ABVD & 19 & 18.7 \\
\hline ABVD-COPP & 14 & 13.9 \\
\hline MOPP & 3 & 2.9 \\
\hline Not received & 7 & 6.8 \\
\hline
\end{tabular}

LDH: Lactate dehydrogenase; COPP: cyclophosphamide, vincristin, procarbazine and prednizone; ABVD: doxorubisin, bleomycin, vinblastine and dacarbazine; ABVD-COPP: doxorubisin, bleomycin, vinblastine and dacarbazinecyclophosphamide, vincristin, procarbazine and prednizone; MOPP: mechlorethamine hydrochloride, vincristine sulfate (oncovin), procarbazine hydrochloride, prednizone. 
Fourteen patients (14\%) had "bulky disease". The disease was mainly localized in cervical lymph nodes, as observed in 96 patients.

The most common subtype was mixed cellularity with 63 patients, while the other subtypes were nodular sclerosis, lymphocytic predominance, lymphocytic depletion, nodular lymphocyte predominant, and unclassified with $12,11,3,2$, and 11 patients, respectively. Fiftynine $(58 \%)$ received COPP, $19(19 \%)$ received
ABVD, 14 patients (14\%) received alternated ABVD-COPP, three patients $(3 \%)$ received MOPP (mechlorethamine hydrochloride, oncovin, procarbazine hydrochloride, prednisolone) chemotherapy and seven patients $(6 \%)$ did not receive any chemotherapy. Eighty-eight patients received involved field radiotherapy. We had two patients that had secondary thyroid carcinoma within the radiotherapy field.

The rates of OS and EFS rates were $89.7 \%$

FIGURE 2. Rates of overall survival and event-free survival

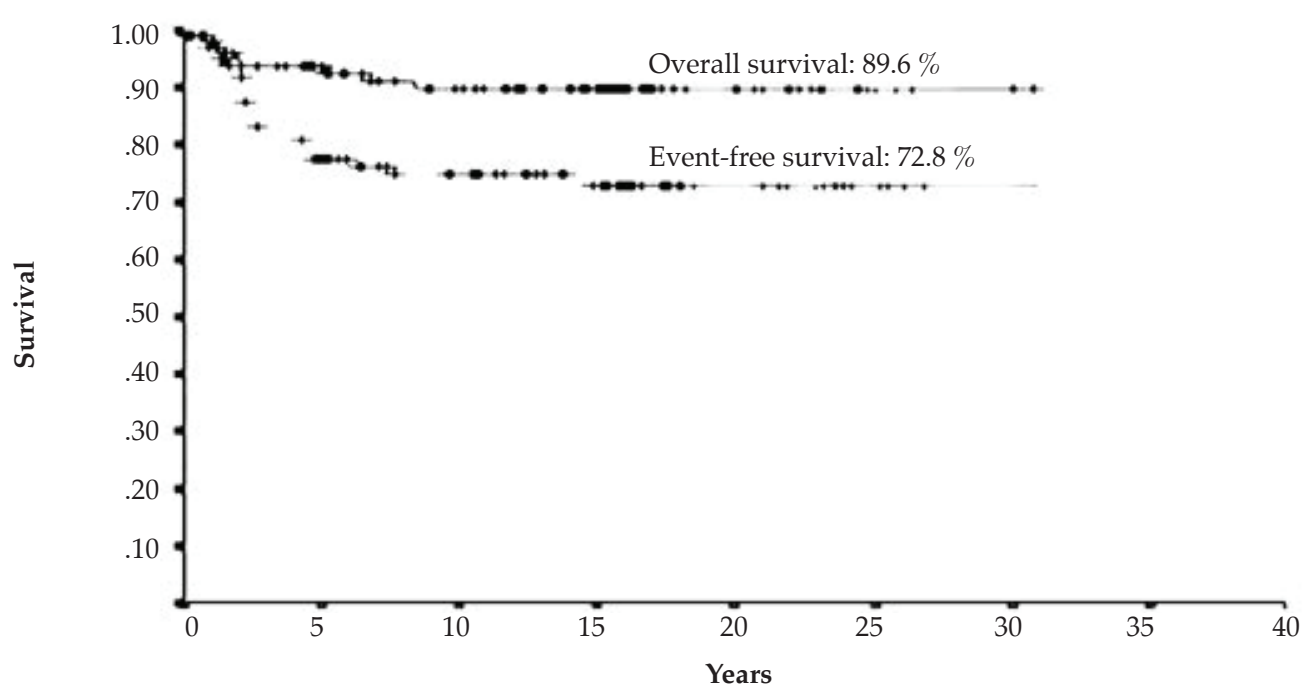

TABLE 2. Event-free and overall survival rates according to stage, chemotherapy, years, B symptoms and blood parameters

\begin{tabular}{|c|c|c|c|c|c|}
\hline & & EFS & $\mathrm{p}$ & os & $\mathrm{p}$ \\
\hline SR & $\begin{array}{l}\text { High } \\
\text { Normal }\end{array}$ & $\begin{array}{l}82.3 \\
94.7\end{array}$ & 0.005 & $\begin{array}{c}96.8 \\
100\end{array}$ & 0.037 \\
\hline Anemia & $\begin{array}{l}\text { Positive } \\
\text { Negative }\end{array}$ & $\begin{array}{l}64.7 \\
76.6\end{array}$ & 0.245 & $\begin{array}{l}76.5 \\
94.5\end{array}$ & 0.023 \\
\hline Period of years & $\begin{array}{l}1970-1980 \\
1980-1990 \\
1990-2000 \\
2000-2013\end{array}$ & $\begin{array}{l}45.8 \\
65.9 \\
86.2 \\
90.9\end{array}$ & 0.01 & $\begin{array}{c}81.8 \\
86.8 \\
92.9 \\
100\end{array}$ & 0.430 \\
\hline B symptoms & $\begin{array}{l}\text { Positive } \\
\text { Negative }\end{array}$ & $\begin{array}{c}53.3 \\
78\end{array}$ & 0.018 & $\begin{array}{l}77.8 \\
92.9\end{array}$ & 0.071 \\
\hline $\mathrm{LDH}$ & $\begin{array}{l}\text { High } \\
\text { Normal }\end{array}$ & $\begin{array}{l}41.1 \\
93.8\end{array}$ & 0.009 & $\begin{array}{c}93.3 \\
100\end{array}$ & 0.129 \\
\hline Stage & $\begin{array}{l}\text { I } \\
\text { II } \\
\text { III } \\
\text { IV }\end{array}$ & $\begin{array}{l}68.1 \\
79.2 \\
68.1 \\
37.5\end{array}$ & 0.051 & $\begin{array}{l}95.1 \\
85.9 \\
84.4 \\
83.3\end{array}$ & 0.540 \\
\hline Chemotherapy & $\begin{array}{c}\text { None } \\
\text { COPP } \\
\text { ABVD } \\
\text { ABVD-COPP } \\
\text { MOPP }\end{array}$ & $\begin{array}{c}42.8 \\
68.5 \\
93.7 \\
82.5 \\
50\end{array}$ & 0.02 & $\begin{array}{c}71.4 \\
88 \\
100 \\
92.8 \\
100\end{array}$ & 0.22 \\
\hline
\end{tabular}

SR: sedimentation rate; LDH: lactate dehydrogenose; EFS: event-free survival; OS: overall survival. 
and $74.8 \%$, respectively (Figure 2). The median follow-up time was 13 years. The rate of OS was $97.0 \%$ in the patients with stage Ia disease, while it was $75 \%$ in patients with stage IVa disease. The rates of OS varied significantly with the stage of disease $(p=0.008)$. The rates of OS in patients with lower hemoglobin levels (under $10 \mathrm{~g} / \mathrm{dl}$ ) was $76 \%$, while it was $94 \%$ in patients with normal hemoglobin levels $(\mathrm{p}=0.023)$ (Table 2). The other significant prognostic factor was sedimentation rate $(\mathrm{p}=0.037)$. Although not statistically significant; the presence of "bulky disease" and B symptoms were associated with poor prognosis. OS rates were $77 \%$ and $91.9 \%$ in patients with and without "bulky disease", respectively $(\mathrm{p}=0.09)$, while they were $77.9 \%$ and $92.9 \%$ in patients with and without B symptoms $(\mathrm{p}=0.07)$.

The prognostic factors affecting the rates of the EFS, were B symptom positivity $(\mathrm{p}=0.018)$, high sedimentation rate $(p=0.005)$, high LDH levels $(\mathrm{p}=0.009)$, and stage IV disease $(\mathrm{p}=0.05)$.

It is also observed that chemotherapy protocols were affecting the event-free survival rates. ABVD based protocols had a significantly positive effect on event free survival $(\mathrm{p}=0.02)$.

Years period have effect on event-free survival; EFS was $90 \%$ during the last decade, while it was $45 \%$ between the 1970 and 1980 period $(\mathrm{p}=0.01)$. OS has gradually increased over the years (from $81 \%$ during the first period to $100 \%$ during the last decade), although the increase was not statistically significant $(\mathrm{p}=0.4)$.

In multivariate analysis, $\mathrm{B}$ symptom positivity $(\mathrm{p}=0.006)$ and years period $(\mathrm{p}=0.022)$ were observed to significantly affect the prognosis.

We did not see any immunodeficiency in this age group.

\section{DISCUSSION}

Our study is one of the most comprehensive single center studies in a developing country to evaluate the clinical characteristics and outcomes of HL in patients under five years of age. This is a retrospective study that spans over three decades, although diagnostic tools and treatment strategies have changed during this period.

Hodgkin's lymphoma occurs with less frequency during childhood and the disease is very rare among children under five years of age. The incidence rate of $14 \%$ observed in our study is consistent with other previously published studies. . $3,6,7$ The incidence of HL in patients under five years of age was observed to gradually decrease over the period of our study.
As documented in our previous report, between 1971 and 1980, $10 \%$ of the patients were under five years old, between 1981 and 1990 period the incidence was $18 \%$, and after the year 2000 it was $5 \%$. ${ }^{7}$ This change could be attributed to improvement of the socioeconomical status of our country. $2,3,7,8$

In previously published studies from our country male-female ratio ranged from 2.25 to 3 . In other studies that have ben done in developed countries, this ratio ranged from 0.7 to 1.6. In our study, the male-female ratio was 3.8:1 which was higher than the ratios documented before..$^{2,7,9}$ The disease was most commonly localized in cervical lymph nodes $(89.5 \%)$, which was similar to other published studies. ${ }^{3.810,11}$ Anemia, high level of lactate dehydrogenase, sedimentation rate, and increased white blood cell count correlated with lower survival rates, which was consistent with other studies published. ${ }^{9,11}$ Twenty-two patients $(22 \%)$ displayed systemic B symptoms and the OS rates were observed to be significantly lower in such patients $(\mathrm{p}=0.01)$. Previously published studies, reported a higher incidence of B symptoms, which was associated with lower survival rates accross all age groups. ${ }^{2,9,11}$ Bulky disease was present in 15 of the 102 patients $(14.7 \%)$ in our study, which was slightly lower than reported in previously published studies across in all age groups. The presence of bulky disease correlated with lower survival rates. ${ }^{7,12-14}$ Bulky disease is one of the worst prognostic factors in patients with Hodgkin lymphoma.

The most common histopathological subtype among our patients was mixed cellularity $(62.3 \%)$. The survival rates did not significantly vary with the histopathological subtype. This finding was comparable with other studies done in our country, on patients in both early and late childhood. ${ }^{2,5}$ In developed countries, nodular sclerosis was the predominant histopathological subtype, whereas in developing countries mixed cellularity was still the most common subtype across age groups. ${ }^{13-17}$ As reported in previously published studies, no statistically significant correlation between the survival rates and histopathological subtypes were found. ${ }^{11,17,18}$ The predominance of mixed cellularity in early childhood, as observed in developing countries including ours, could be linked with the early exposure to Epstein-Barr virus or with other carcinogenic factors. We had two patients with nodular lymphocyte predominant histopathology. They were treated with chemotehrapy and radiotherapy. However, for the last 5 years, 
treatment options according to the stage were: observation without any treatment, rituximab alone or rituximab combined with chemotherapy respectively.

Twenty-five patients had stage III-IV disease, while 77 had stage I and II disease. The incidence of advanced stages was observed to be lower, which was consistent with other studies carried out on similar age groups. Although the stage of disease did not significantly affect the OS, the rates of OS were higher in early stages of the disease. Event-free survival rates were higher in early stages $(p=0.05)$. The previously published studies from our country reported early stages to have higher incidence and survival rates than advanced stages among children with HL.7,9,12 In contrast with our study, one study from our country reported a higher incidence of advanced stages among children under six years of age. ${ }^{2}$

Our general strategy to treat patients has been the use of a combination of two to four cycles of chemotherapy with low dose radiotherapy in early stages and the combination of four to six cyles of chemotherapy with radiotherapy in advanced stages. We had two patients with secondary thyroid carcinoma within the radiotherapy field.

This retrospective study spans three decades of research at our center. The median followup time of 13 years is one of the longest periods reported in the literature. The OS and EFS rates were $89.7 \%$ and $74.8 \%$, respectively. A previous study conducted by Stoneham et al., ${ }^{3}$ on children under 5 years of age, reported the rate of OS for stages II-IV as $89 \%$, and emphasized that the advanced stages at presentation were the only predictors for prognosis. Belgaumi et al., ${ }^{6}$ reported OS rate of $90.4 \%$ over a 10 years period in children under 5 years of age. EFS rate of $74.8 \%$ was comparable with other studies that were previously published. Stoneham et al. reported EFS rates of $88 \%$ and $68 \%$ from two trials conducted during different time periods, while Belgaumi et al. reported EFS rate of $81.5 \%$ for children under 5 years of age. $., 7,8,14,19$ Moreover, in a recent study by Farrugia et al, though the EFS rate was lower than the present study, OS rate $(97 \%)$ at 10 years for patients under seven years of age was remarkably higher than the present study. ${ }^{4}$

Univariate analysis of the data indicated that high sedimentation rates and the presence of anemia were linked to decreased OS rates.

In summary, this study is one of the most comprehensive single center studies in a developing country that spans over three decades to evaluate the clinical characteristics and outcomes of HL in patients under five years of age. Although the diagnostic tools and treatment strategies have changed over this long period of time, our findings indicated that the stage of disease and B symptoms were the only predictors for rates of OS and EFS. The trends in these rates were similar to those reported in other studies conducted on patients younger than 5 years and 18 years of age. It is worth consideration by oncologists that this age group has a longer time of remaining life than other age groups. Therefore, the treatment strategy should aim at maintaining the superior OS rates while minimizing the treatment related toxicity for children under 5 years of age. The patients with poor prognostic factors should be treated with different strategies.

\section{REFERENCES}

1. Capra M, Hewitt M, Radford M, Hayward J, et al. Longterm outcome in children with Hodgkin's lymphoma: the United Kingdom Children's Cancer Study Group HD82 trial. Eur J Cancer. 2007; 43(7):1171-9.

2. Cavdar AO, Gozdasoglu S, Yavuz G, Unal E, et al. Characteristics of "Early Type-I Pattern" (0-6 years) Hodgkin's Disease in Turkish Children. Turk J Haematol. 2002; 19(1):55-62.

3. StonehamS, AshleyS, Pinkerton R, HewittM, etal.Hodgkin's lymphoma in children aged 5 years or less - the United Kingdom experience. Eur J Cancer. 2007; 43(9):1415-21.

4. Farruggia P, Puccio G, Locatelli F, Vetro M, et al. Classical pediatric Hodgkin lymphoma in very young patients: the Italian experience. Leuk Lymphoma. 2019; 60(3):699-702.

5. Metzger M, Krasin MJ, Hudson MM, Onciu M. Hodgkin Lymphoma. In: Pizzo A, Poplack D (eds). Principles and Practice of Pediatric Oncology. 6th ed. Philadelphia: Lippincott Williams and Wilkins; 2011.Págs.639-61.

6. Belgaumi AF. Hodgkin's lymphoma in the young child. Transfus Apher Sci. 2010; 42(2):163-7.

7. Büyükpamukçu M, Varan A, Akyüz C, Atahan L, et al. The treatment of childhood Hodgkin lymphoma: improved survival in a developing country. Acta Oncol. 2009; 48(1): 44-51.

8. Belgaumi A, Al-Kofide A, Joseph N, Jamil-Malik R, et al. Hodgkin lymphoma in very young children: Clinical characteristics and outcome of treatment. Leuk Lymphoma. 2008; 49(5):910-6.

9. Oguz A, Karadeniz C, Okur FV, Citak EC, et al. Prognostic factors and treatment outcome in childhood Hodgkin disease. Pediatr Blood Cancer. 2005; 45(5):670-5.

10. Ali A, Sayed H, Farrag A, El-Sayed M. Risk-based combinedmodality therapy of pediatric Hodgkin's lymphoma: a retrospective study. Leuk Res. 2010; 34(11):1447-52.

11. Arya LS, Dinand V, Thavaraj V, Bakhshi S, et al. Hodgkin's disease in Indian children: outcome with chemotherapy alone. Pediatr Blood Cancer. 2006;46(1):26-34.

12. Büyükpamukçu M, Atahan L, Caglar M, Kutluk T, et al. Hodgkin's disease in Turkish children: clinical characteristics and treatment results of 210 patients. Pediatr Hematol Oncol. 1999; 16(2):119-29. 
13. Nachman JB, Sposto R, Herzog P, Gilchrist GS, et al. Randomized comparison of low-dose involved-field radiotherapy and no radiotherapy for children with Hodgkin's disease who achieve a complete response to chemotherapy. J Clin Oncol. 2002; 20(18):3765-71.

14. Kulyova SA, Kolygin BA. Hodgkin's Lymphoma in Children and Adolescents: A Saint Petersburg Hodgkin's Lymphoma Group Study. J Oncol. 2011; 2011:958435.

15. Tebbi CK, Mendenhall NP, London WB, Williams JL, et al. Response-dependent and reduced treatment in lower risk Hodgkin lymphoma in children and adolescents, results of P9426: a report from the Children's Oncology Group. Pediatr Blood Cancer. 2012; 59(7):1259-65.

16. Hakvoort-Cammel FG, Buitendijk S, van den HeuvelEibrink M, Hahlen K. Treatment of pediatric Hodgkin disease avoiding radiotherapy: excellent outcome with the Rotterdam-HD-84-protocol. Pediatr Blood Cancer. 2004; 43(1):8-16.

17. Hsu SC, Metzger ML, Hudson MM, Pedrosa F, et al. Comparison of treatment outcomes of childhood Hodgkin lymphoma in two US centers and a center in Recife, Brazil. Pediatr Blood Cancer. 2007; 49(2):139-44.

18. Bazzeh F, Rihani R, Howard S, Sultan I. Comparing adult and pediatric Hodgkin lymphoma in the Surveillance, Epidemiology and End Results Program, 1988-2005: an analysis of 21734 cases. Leuk Lymphoma. 2010; 51(12): 2198-207.

19. Wolden SL, Chen L, Kelly KM, Herzog P, et al. Longterm results of CCG 5942: a randomized comparison of chemotherapy with and without radiotherapy for children with Hodgkin's lymphoma--a report from the Children's Oncology Group. J Clin Oncol. 2012; 30(26):3174-80. 